\title{
THE MAGNETIC STATE OF THE LOWER IONOSPHERE DURING PIONEER VENUS ENTRY PHASE
}

\author{
C. T. Russell, R. J. Strangeway and J. G. Luhmann \\ Institute of Geophysics and Planetary Physics, University of California, Los Angeles
}

L. H. Brace

Space Research Laboratory, University of Michigan, Ann Arbor, Michigan

\begin{abstract}
During the entry phase of the Pioneer Venus Orbiter, defined as that period at the end of mission in 1992 when the periapsis fell below $185 \mathrm{~km}$, the magnetometer made repeated measurements throughout the post midnight ionosphere until about 0430 LT. In this region the magnetic field is generally stronger at comparable altitudes than it was earlier at times of higher solar activity. This increase combined with a decrease in electron density causes the ratio of the magnetic pressure to thermal pressure to approach unity at altitudes above $200 \mathrm{~km}$, whereas it was much lower than unity at these altitudes during solar maximum. From $160-200 \mathrm{~km}$ the magnetic field pressure exceeds that of the ionospheric plasma quite unlike the usual conditions seen at the beginning of the mission. At lowest altitudes below 150 $\mathrm{km}$, however, the field becomes weaker and hence no evidence for a planetary magnetic field is found.
\end{abstract}

\section{Introduction}

The earlier measurements of the Pioneer Venus mission revealed the properties of the ionosphere and its dependence on solar and solar wind conditions at altitudes above $150 \mathrm{~km}$ and for solar activity near its maximum [Luhmann and Cravens, 1991; Russell and Vaisberg, 1983]. At solar maximum the Venus ionosphere had sufficient thermal pressure to stand off the solar wind well above the atmosphere at times of normal solar wind dynamic pressure and was basically magnetic field free, but when the solar wind pressure became high, the ionosphere became magnetized. The night ionosphere was just as sensitive to this solar wind control as the dayside, because at solar maximum the dayside ionosphere supplied the bulk of the nightside ionization observed above about $200 \mathrm{~km}$ altitude through flow across the terminators. Features known as ionospheric "holes" [Brace et al., 1982] with enhanced nearly vertical fields within were regularly observed at these times. When the solar wind pressure was high, this nightward transport was ineffective and the condition known as the "disappearing ionosphere" prevailed [Cravens et al., 1982]. Disappearing ionospheres were accompanied by enhanced ( $20-40 \mathrm{nT})$ horizontal fields that dominated the pressure above $-160 \mathrm{~km}$ altitudes.

As the mission progressed, it became clear that as the solar cycle changed, so did the ionosphere. One of the most

\section{Copyright 1993 by the American Geophysical Union.}

Paper number 93GL02625

0094-8534/93/93GL-02625\$03.00 dramatic effects was the change in the average location of the bow shock [Russell et al., 1988]. The shrinking of the bow shock at solar minimum was attributed to a weakening of the ionosphere related to the decreasing solar EUV flux. Radio occultation data at solar minimum confirmed this interpretation [Knudsen et al., 1987], but no in situ ionospheric data were available at low solar activity conditions because periapsis had moved to very high altitudes. Thus, the entry phase data were long anticipated as they would provide low altitude measurements under solar conditions quite different than those available early in the mission when the first low altitude data were acquired. Furthermore, the entry phase data would reach altitudes significantly below those achieved in the early mission. Figure 1 shows the periapsis altitude on the entry phase orbits that we will be considering in this letter. Local time is shown decreasing from right to left. This figure illustrates the view of successive periapses as seen looking down on the planet from the wake region. The last available measurements were obtained on orbit 5055 .

The objective of this study is to examine the magnetic field and plasma pressures at lowest altitudes and to compare their behavior with that seen earlier in the mission under conditions of high solar activity. Since the spacecraft did not

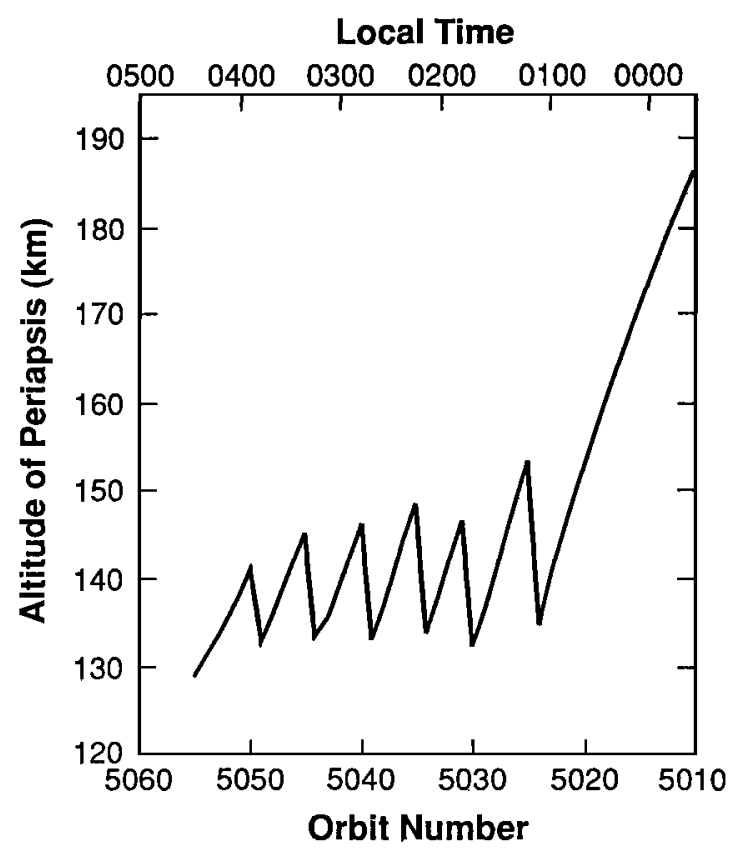

Fig. 1. Altitude of periapsis versus local time and orbit number during the entry phase of the Pioneer Venus mission. 
reach low altitudes until it approached midnight local time, our study will be applicable only to the post-midnight or early morning hours in the night ionosphere.

\section{The Magnetic Field}

Comparisons of the magnetometer entry data with data obtained earlier in the mission are complicated by the apparent failure of a multiplexer in October 1988. After this time, the spin axis sensor is sampled three times per minor frame rather than each of the sensors being sampled once. This sensor measures the field perpendicular to the orbital plane of Venus. Furthermore, toward the end of the mission, the zero level of this sensor began to drift slowly. Nevertheless, as we demonstrate below, we can determine useful information on the statistical ionospheric magnetic field even if we do not know the instantaneous value of all 3 components of the magnetic field.

We proceed by first finding the median spin axis component of the magnetic field over all the entry phase which we take here to be orbits 5011 to 5055 . Over this period we expect the magnetic field in the night ionosphere to vectorially sum to zero. This median field of $26 \mathrm{nT}$ we take to be the offset of the magnetometer. We use a median rather than an average to mitigate the effect of outlying points. We remove this median field from the measurements and then rectify the magnetic field i.e., take its absolute value. We then perform our statistical analyses on the rectified data. The median value, upper quartile and maximum value as a function of altitude up to $300 \mathrm{~km}$ are shown in Figure 2. The magnitude of this component of the field reaches its largest values at about $180 \mathrm{~km}$ in the median and upper quartile values and at about $195 \mathrm{~km}$ in the maximum field. Below $170 \mathrm{~km}$ this field decreases rapidly with decreasing altitude. During entry phase the average sunspot number was 64 and the average $F 10.7 \mathrm{~cm}$ flux at 1 AU was 117.

The magnetic field behavior is different than during typical solar maximum conditions. Figure 3 shows the results of an

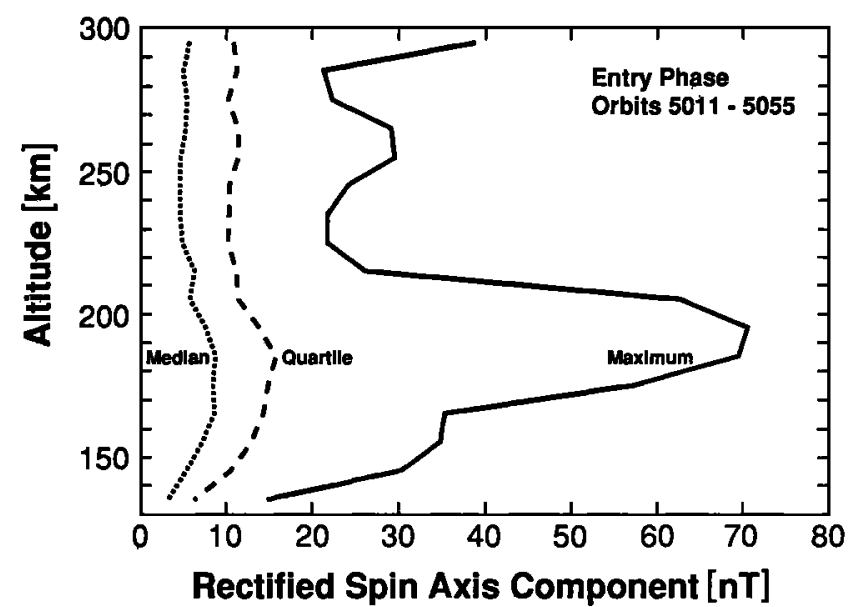

Fig. 2. Altitude profile of the rectified spin axis magnetic field during the PVO entry phase. Shown are the median, upper quartile and maximum field encountered in each $10 \mathrm{~km}$ altitude bin. A zero level field has been removed from these data. The zero level was found using medians of the vector component over the entry phase. identical analysis using the rectified spin axis component of the magnetic field for orbits 521-560 in season 3 when periapsis was maintained at low altitudes. During this interval, the average sunspot number was 180 and the average $10.7 \mathrm{~cm}$ flux at $1 \mathrm{AU}$ was 224 . At solar maximum the median spin axis-aligned magnetic field is lower than during the entry phase at all altitudes above $165 \mathrm{~km}$ and higher at all altitudes below $165 \mathrm{~km}$. Both the upper quartile magnetic field and the maximum magnetic field behave similarly. This change is not due to a change in calibration of the magnetometer. A calibrate winding is included around each sensor and was exercised throughout the mission. No measurable gain change occurred between the laboratory calibration and the end of mission. It is clear that as solar activity declined from solar maximum to moderate solar activity conditions, the magnetic field increased at higher altitudes and decreased at lower altitudes. Furthermore, the magnetic field was weakest at lowest altitudes under these moderate solar conditions.

\section{Ionospheric Pressure}

In order to understand the dynamics of the ionosphere, we need to understand the relative importance of the magnetic and thermal pressures. Since we have only one magnetic sensor during the entry phase, we resort to statistical arguments to do this. Figure 4 shows the magnetic field strength from season 3 orbits $521-560$ in the same format as the rectified spin axis component in Figure 2 and 3. Figure 5 shows the correlation between these values in each $10-\mathrm{km}$ altitude bin with the spin axis component in Figure 3 . The correlation is quite good and suggests that by using a factor of 2.5 , we can convert statistically the field component measurements to field magnitudes. It is notable that when this factor is applied to the medians in Figure 2, field strengths the order of those observed in the disappearing ionosphere situations [e.g., Luhmann, 1992] result.

In order to approximate the plasma thermal pressure, we assume the ion and electron temperatures are equal to the observed electron temperature. We note that much of our data is at low altitudes in the collisional regime where we

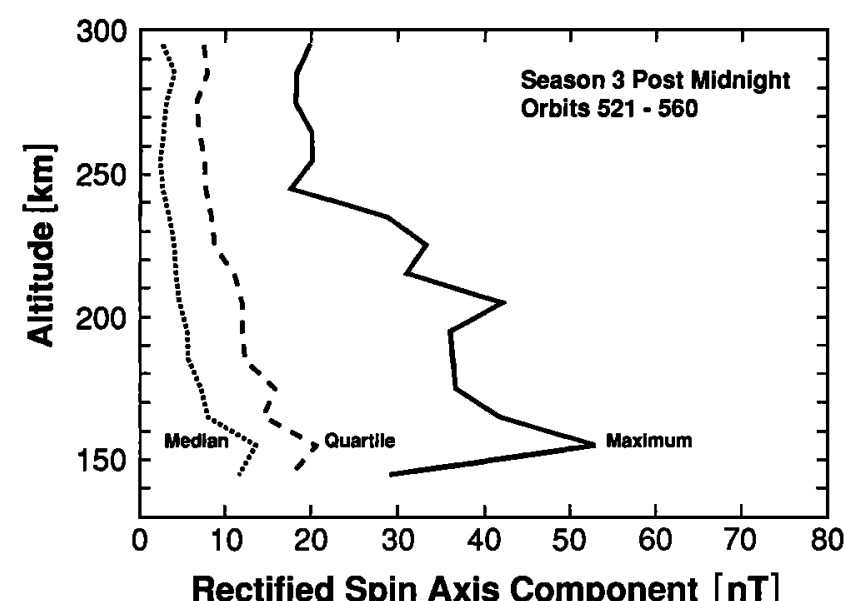

Fig. 3. Altitude profile of the rectified spin axis magnetic field during the post midnight period of season 3 corresponding to the entry phase data. Median, upper quartile and maximum fields are shown. 


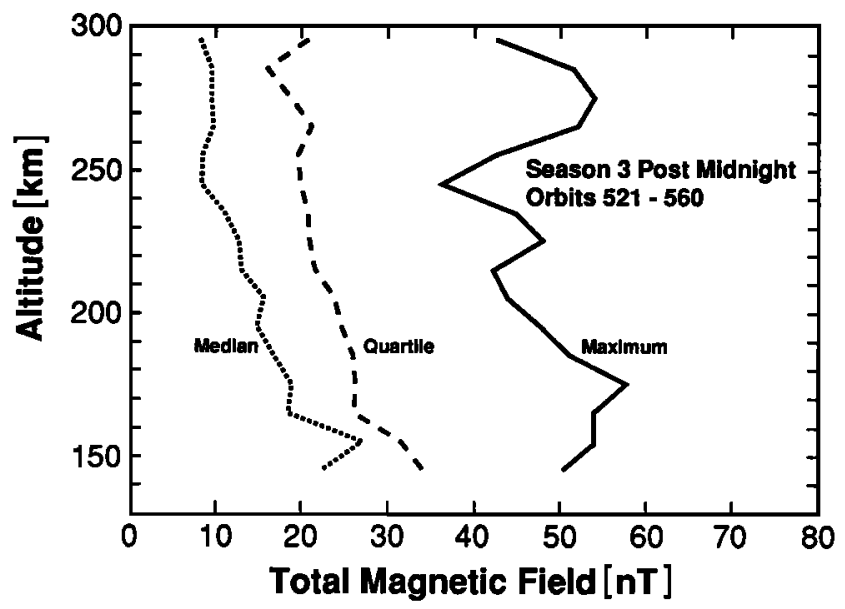

Fig. 4. Altitude profile of the total magnetic field in the postmidnight sector during season 3. Median, upper quartile and maximum fields are shown.

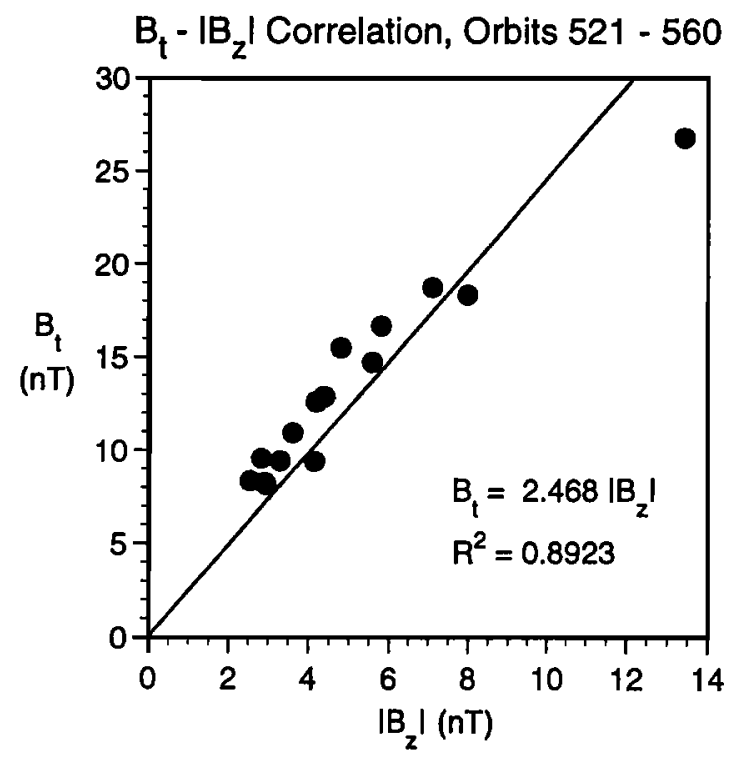

Fig. 5. The correlation between the total magnetic field and the spin axis $\left(B_{z}\right)$ component in the postmidnight sector of season three. Each point represents the median value of $B_{t}$ and $B_{z}$ in a $10 \mathrm{~km}$ altitude bin. The slope of the correlation line is 2.5 .

expect the ion and electron temperatures to be equal. In fact nighttime ion temperatures differ only about $20 \%$ from electron temperatures below $300 \mathrm{~km}$ [Miller et al., 1980] with the difference going to zero at lowest altitudes. Making this assumption, we get the profiles seen in Figures 6 and 7 . During season 3 at a time of high solar activity, the magnetic pressure is much less than that of the plasma at all altitudes in the $140 \mathrm{~km}$ to $300 \mathrm{~km}$ range. At the moderate solar activity of the entry phase, this thermal pressure drops markedly and the magnetic pressure increases (at the higher altitudes). Hence, during the entry phase above $200 \mathrm{~km}$, the ionosphere has a beta (the ratio of thermal to magnetic pressure) of close to unity. From 160 to $200 \mathrm{~km}$, beta is less than 1. Thus, above $160 \mathrm{~km}$ at times of moderate to low solar activity, the magnetic field is important in determining the ionospheric plasma dynamics. At lowest altitudes the

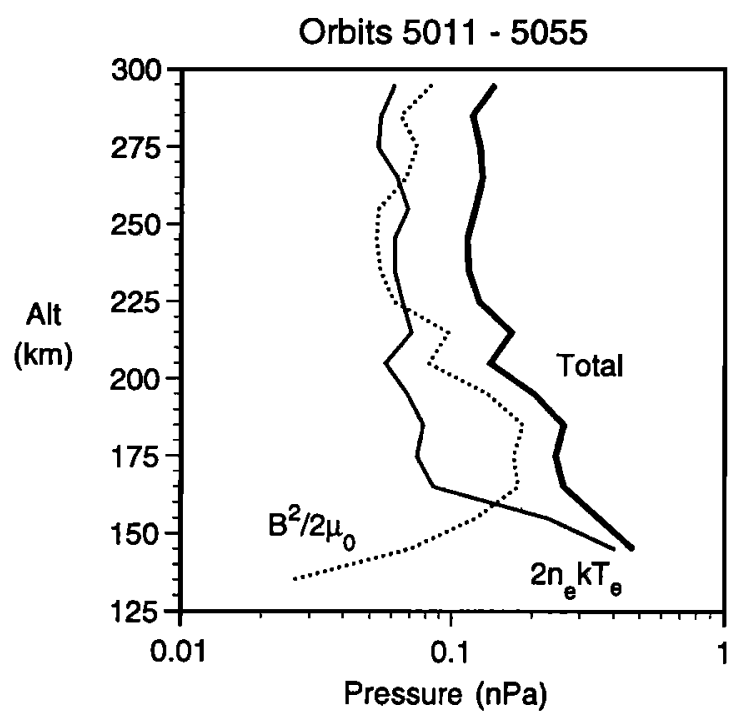

Fig. 6. The altitude profile of magnetic and thermal pressure in the ionosphere during the entry phase. The magnetic field magnitude has been obtained at each altitude by multiplying the median rectified spin axis component by a factor of 2.5 . The ionosphere thermal pressure has been obtained by using the median electron density at each altitude, multiplying by the temperature profile observed during season 3 , and doubling to account for the ion pressure. The electron data below $140 \mathrm{~km}$ are not shown since effects due to the interaction of the spacecraft with the atmosphere have not yet been removed.

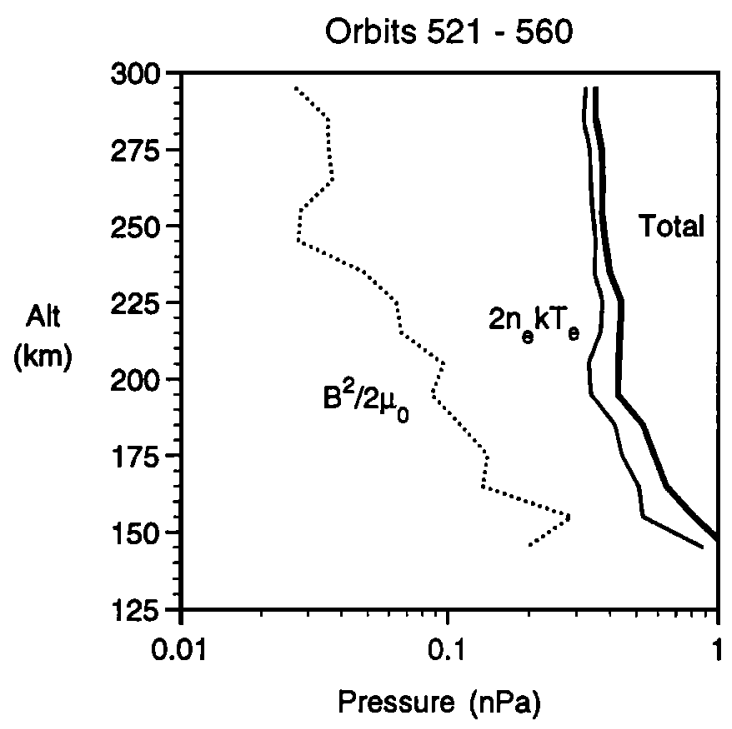

Fig. 7. The altitude profile of the magnetic and thermal pressure in the ionosphere in the postmidnight sector during season 3. The total thermal pressure has been obtained assuming that the ion and electron temperatures are equal.

magnetic pressure drops markedly, and the plasma pressure becomes dominant. This behavior is similar to those disappearing ionospheres which are "weakly" disappearing. In the most depleted nightside ionosphere cases (e.g., those described by Luhmann [1992]), the magnetic pressure exceeds the plasma pressure everywhere above $-160 \mathrm{~km}$ by up to an order of magnitude. 
Summary and Conclusions

We have examined the altitude profiles of the magnetic field in the low altitude nightside ionosphere of Venus obtained during Pioneer Venus orbiter entry and compared them with earlier observations. These studies show that above about $160 \mathrm{~km}$ the magnetic field generally became stronger when the solar activity decreased. Since the electron density became weaker and the temperature was only slightly greater at times of low solar activity, the net result was that the magnetic pressure equaled or dominated the thermal pressure above about $160 \mathrm{~km}$ altitude. In contrast at times of high solar activity the thermal pressure dominated the magnetic pressure up to at least $300 \mathrm{~km}$. We expect that when solar activity dropped even further, later in the solar cycle, the magnetic field would exercise even more control over the dynamics of the nightside ionosphere. The observed intermediate solar activity conditions resemble the weaker of the disappearing ionospheres seen at solar maximum, consistent with the idea that at solar minimum fully developed disappearing ionospheres prevail [e.g., Knudsen et al., 1987; Zhang et al., 1990].

Data obtained at the very bottom of the nightside ionosphere (near $130 \mathrm{~km}$ ) is interesting because it could lead to better information about the possibility of internal sources contributing to the observed magnetic field. At times of high solar activity, a slight fall-off in field strength was seen from 165 to $140 \mathrm{~km}$, the lowest altitude reached. At moderate solar activity during the entry phase, the low altitude magnetic field decreased rather sharply below $165 \mathrm{~km}$ altitude down to $128 \mathrm{~km}$, the minimum altitude from which data were returned. Thus, these data provide no evidence in support of the existence of a planetary magnetic moment. The planet appears to have by far the lowest magnetic moment of any planet visited to date [Russell et al., 1980; Phillips and Russell, 1987].

Acknowledgments. We would like to thank the many individuals who helped make Pioneer Venus a most resounding success. In particular, we wish to acknowledge the efforts of the following individuals during the entry phase: L. Colin, J. Dyer, R. Fimmel, R. Jackson, L. Lasher, D. Lozier and M. Smith. This work was supported by the National Aeronautics and Space Administration under research grants NAG 2-501, NAG 2-485 and NAGW-3492.
References

Cravens, T. E., L. H. Brace, H. A. Taylor Jr., C. T. Russell, W. L. Knudsen, K. L. Miller, A. Barnes, J. D. Mihalov, F. L. Scarf, S. J. Quenon, and A. F. Nagy, Disappearing ionospheres on the nightside of Venus, Icarus, 51, 271282, 1982.

Knudsen, W. C., A. J. Kline and R. C. Whitten, Solar cycle changes in the ionization sources of the nightside Venus ionosphere, J. Geophys. Res., 92, 13,391-13,398, 1987.

Luhmann, J. G., Pervasive large-scale magnetic fields in the Venus nightside ionosphere and their implications, $J$. Geophys. Res., 97, 6103-6121, 1992.

Luhmann, J. G., and T.A. Cravens, Magnetic fields in the ionosphere of Venus, Space Sci. Rev., 55, 201-274, 1991.

Miller, K. L., W. C. Knudsen, K. Spenner, R. C. Whitten and V. Novak, Solar zenith angle dependence of ionospheric ion and electron temperatures and density on Venus, $J$. Geophys. Res., 85, 7759-7764, 1980.

Phillips, J. L., and C. T. Russell, Upper limit on the intrinsic magnetic field of Venus, J. Geophys. Res., 92, 2253-2263, 1987.

Russell, C. T., and O. L. Vaisberg. The interaction of the solar wind with Venus, in Venus, pp. 873-940, University of Arizona Press, Tucson, 1983.

Russell, C. T., R. C. Elphic, J. G. Luhmann, and J. A. Slavin, On the search for an intrinsic magnetic field at Venus, Proc Lunar Planet Sci Conf, 11th, pp. 1897-1906, 1980.

Russell, C. T., E. Chou, J. G. Luhmann, P. Gazis, L. H. Brace, and W. R. Hoegy, Solar and interplanetary control of the location of the Venus bow shock, J. Geophys. Res., 93, 5461-5469, 1988.

Zhang, M.H.G., J. G. Luhmann, and A. J. Kliore, An observational study of the nightside ionospheres of Mars and Venus with radio occultation methods, J. Geophys. Res., 95, 17,095-17,102, 1990.

L. H. Brace, Space Research Laboratory, University of Michigan, Ann Arbor, Michigan 48105.

C. T. Russell, R. J. Strangeway and J. G. Luhmann, Institute of Geophysics and Planetary Physics, University of California, Los Angeles, CA 90024-1567.

(Received July 1, 1993;

revised August 16, 1993; accepted September 1, 1993) 